\title{
New insights in Trypanosoma cruzi proteomic map: further post- translational modifications and potential drug targets in Y strain epi- mastigotes
}

\author{
Daniela Gois Beghini ${ }^{1}$, André Teixeira da Silva Ferreira ${ }^{1}$, Vivian Corrêa de Almeida ${ }^{2}$, Marcelle Almeida Caminha ${ }^{3}$, Floriano \\ Paes Silva-Jr², Jonas Perales ${ }^{1}$, Rubem Figueiredo Sadok Menna-Barreto ${ }^{\star 3}$
}

${ }^{1}$ Laboratório de Toxinologia, Instituto Oswaldo Cruz, Fundação Oswaldo Cruz, Rio de Janeiro, Brazil; ${ }^{2}$ Laboratório de Bioquímica de Proteínas e Peptídeos, Instituto Oswaldo Cruz, Fundação Oswaldo Cruz, Rio de Janeiro, Brazil; ${ }^{3}$ Laboratório de Biologia Celular, Instituto Oswaldo Cruz, Fundação Oswaldo Cruz, Rio de Janeiro, Brazil.

Received: 24 August 2012 Accepted: 03 November 2012 Available Online: 09 November 2012

\section{Abstract}

\begin{abstract}
Chagas' disease is a neglected sickness endemic in Latin America, caused by the protozoa Trypanosoma cruzi. The current treatment for the disease is unsatisfactory, and the development of potent compounds for novel molecular targets is critical. In this framework, proteomics could be a powerful tool in the evaluation of possible candidates for drug intervention. In this work, a two-dimensional electrophoresis (2$\mathrm{DE}$ ) and mass spectrometry (MS) approaches were employed in T. cruzi epimastigotes (Y strain). Different gel staining protocols (Coomassie Blue, Pro-Q-Diamond and Pro-Q-Emerald) were performed to assess the protein content and possible post-translational modifications of this parasite. Here, 78 most intense spots were identified by Coomassie staining, 22 by Pro-Q-Diamond (phosphoproteins) and 15 by Pro-QEmerald (glycoproteins). Compared with the results of other large-scale T. cruzi proteomic studies, 15 novel proteins were identified here using MALDI-TOF/TOF, and 12 of these have not yet been described at the protein level. Functional analysis of the identified proteins pointed to protein metabolism, and the localisation prediction indicated cytosol as the most prevalent localisation of these proteins. Eight proteins presented no similarity to human sequences and thus represent a group of promising biomolecules for chemotherapy intervention. Our data provides novel insights in the metabolic pathways of T. cruzi, which could aid in the discovery of alternative drugs for Chagas' disease.
\end{abstract}

Keywords: Trypanosoma cruzi; Chagas' disease; Chemotherapy; Post-translational modifications; Mass spectrometry; Proteomics .

\section{Introduction}

Chagas' disease is a neglected tropical disease that is endemic in Latin America and was first reported in an ancient Chinchorro civilisation 9,000 years ago [1]. This illness is caused by infection with its etiological agent, the hemoflagellate protozoan Trypanosoma cruzi. The T. cruzi life cycle involves invertebrate and vertebrate hosts as well as the three evolutive forms of the protozoa. In the triatomine midgut, epimastigotes proliferate and subsequently migrate to the posterior intestine, where differentiation into the infective metacyclic trypomastigote form occurs. After haematophagy, the infective metacyclic forms reach the bloodstream of the vertebrate host where they can infect the cells of various mammalian tissues. In the intracellular environment, the parasites differentiate into proliferative amastigotes. Subsequently, another differentiation occurs to produce trypomastigotes, which are responsible for disseminating the in-

*Corresponding author: Dr. R.F.S. Menna-Barreto, Laboratório de Biologia Celular, Instituto Oswaldo Cruz, FIOCRUZ, Av. Brasil 4365 - 21040-360, Manguinhos, Rio de Janeiro, Brazil; Phone number: 005521 25621393; Fax number: 005521 25621432; E -mail adress: rubemb@ioc.fiocruz.br 
fection. The transmission of Chagas' disease primarily occurs by infection with the triatomine vector, but it also has been reported to result from blood transfusion, congenital transmission, organ transplantation, the ingestion of contaminated food and laboratory accidents [2]. Due to its anthropic action, the triatomine favours a domiciliary and peridomiciliary behaviour, increasing human exposure and risk of infection. A recent report stated that 8 million people in Latin America have this disease and described the major concern that immigration-related globalisation will bring infected people to non-endemic regions, such as Europe, North America and Australia [2].

The disease treatment is still based on benznidazole and nifurtimox, nitroderivatives that were empirically developed forty years ago and present important limitations, such as high doses, severe side effects and controversial efficacy in chronic patients [3]. Intense efforts have been directed to discover new compounds against $T$. cruzi, better characterise their particular mechanisms of action and identify the molecules and pathways involved [4].

Gene expression in trypanosomatids, including T. cruzi, is very unusual in that the open reading frames are arranged in long polycistronic arrays and monocistronic mRNAs are created by post-transcriptional processing. Thus, the regulation of gene expression in $T$. cruzi is mainly posttranscriptional [5]. Previous studies have revealed nontranslated mRNA in the T. cruzi cytoplasm [6]. Therefore, the use of nucleic acid-based tools, such as RNA microarrays and PCR, to study gene expression is limited in trypanosomatids, and thus, the application of proteomic techniques is highly desirable.

Proteomics is a high-throughput approach that is extensively employed in structural and functional studies on the regulation of protein expression to validate genome annotations [7]. Different stage-specific molecules and proteins associated with parasite drug resistance have been identified by proteomic approaches in T. cruzi [8-11], reinforcing the importance of these techniques in the selection of metabolic targets of new inhibitors or therapeutic drugs $[2,12]$.

In the present study, we used two-dimensional electrophoresis (2-DE) and mass spectrometry to contribute additional information to the descriptive proteomic map of T. cruzi epimastigotes ( $Y$ strain). The $Y$ strain is a natural strain of $T$. cruzi I that is found in Brazil and presents high parasitaemia and mortality in mouse models. Despite its virulence, the Y strain is susceptible to benznidazole treatment [13]. Here, $T$. cruzi samples analysed by 2-DE were stained with different dyes to reveal specific phosphoproteins and glycoproteins, which were then identified by MS. The identified T. cruzi glycoproteins are particularly important as targets for therapeutic intervention, and our analysis of the phosphorylated proteins could provide crucial insights into the signalling networks that govern the metabolism of this parasite. The identified peptides and proteins were characterised using bioinformatic tools in an attempt to find potential drug targets.

\section{Materials and methods}

\subsection{Parasites and sample preparation}

T. cruzi epimastigotes ( $\mathrm{Y}$ strain) were maintained in liver infusion tryptose (LIT) medium at $28^{\circ} \mathrm{C}$ and harvested during exponential growth phase. The parasites were harvested by centrifugation, washed three times with phosphate buffered saline (PBS, pH 7.4) and incubated in sample lysis solution (7 M urea, $2 \mathrm{M}$ thiourea, $4 \%$ CHAPS, $40 \mathrm{mM}$ Tris, 60 mM DTT, $1 \%$ ampholytes) containing the Complete Mini protease inhibitor cocktail (Roche Applied Science, Indianapolis, USA). The parasite cells were further disrupted via ten cycles of freezing in liquid nitrogen and thawing at room temperature, with gentle mixing between cycles, as previously described [11]. To obtain the soluble protein fraction, the cell lysate was centrifuged at $13,000 \mathrm{~g}$ for $30 \mathrm{~min}$, and the protein content was determined using the $2 \mathrm{D}$ Quant kit (GE Healthcare, Buckinghamshire, England).

\subsection{Two-dimensional electrophoresis and image analysis}

Isoelectric focusing of the soluble protein fraction from the epimastigote extracts was performed using an IPGphor system (GE Healthcare) and 18-cm IPG strips of $\mathrm{pH} 4-7$. The protein fraction was diluted in rehydration buffer $(7 \mathrm{M}$ urea, $2 \mathrm{M}$ thiourea, 4\% CHAPS, $60 \mathrm{mM}$ DTT, $1 \%$ ampholytes and a trace amount of bromophenol blue) and loaded onto each strip. The strips were rehydrated for $12 \mathrm{~h}$ at $30 \mathrm{~V}$ and isoelectrically focused using the following protocol: $200 \mathrm{~V} / 1 \mathrm{~h}, 500$ $\mathrm{V} / 1 \mathrm{~h}, 1000 \mathrm{~V} / 1 \mathrm{~h}$, a gradient of 1000 to $8000 \mathrm{~V}$ for $10 \mathrm{~min}$ and $8000 \mathrm{~V} / 6 \mathrm{~h}$. The strips were then equilibrated in $50 \mathrm{mM}$ Tris $-\mathrm{HCl}$ (pH 8.8), $6 \mathrm{M}$ urea, 30\% v/v glycerol, $2 \% \mathrm{w} / \mathrm{v}$ SDS, $0.002 \% \mathrm{w} / \mathrm{v}$ bromophenol blue and $1 \% \mathrm{w} / \mathrm{v}$ DTT for $15 \mathrm{~min}$, followed by a second 15-min incubation in the same buffer but with $4 \%$ iodoacetamide instead of DTT. Separation in the second dimension was performed by SDS-PAGE using $12 \%$ acrylamide gels at $2.5 \mathrm{~W} /$ gel for $30 \mathrm{~min}$ and then $100 \mathrm{~W}$ until completion using a DALTsix system (GE HealthCare). .The proteins separated on the $2 \mathrm{D}$ gels were stained to detect phosphoproteins, glycoproteins or total protein as described below.

\subsection{Detection of total protein}

Gels were fixed three times with $30 \%$ ethanol and $2 \%$ phosphoric acid for $30 \mathrm{~min}$ per wash, followed by three successive washes in $2 \%$ phosphoric acid in water for $20 \mathrm{~min}$. Then, the gels were incubated in $18 \%$ ethanol, $2 \%$ phosphoric acid and 15\% ammonium sulphate for $30 \mathrm{~min}$, followed by the addition of $2 \%$ Coomassie Brilliant Blue $G$ in water (Sigma-Aldrich, St. Louis, USA). The gels were scanned and the spots were detected, quantified and analysed with Image Master Platinum software (GE Healthcare). Each spot was manually inspected and, when necessary, edited with the Edit Spot Tools to improve accuracy. 


\subsection{Detection of phosphoproteins}

2D gels were fixed in 50\% methanol and $10 \%$ acetic acid overnight, washed three times with deionised water for 10 min per wash and incubated in Pro-Q Diamond phosphoprotein stain (Molecular Probes) for $90 \mathrm{~min}$. To reduce the background signal, gels were destained with three successive washes of $20 \%$ acetonitrile and $50 \mathrm{mM}$ sodium acetate $(\mathrm{pH}$ 4.0). Images were acquired with a Typhoon Trio system (GE Healthcare) using a $532 \mathrm{~nm}$ laser for excitation and a $580 \mathrm{~nm}$ filter for emission. Following image acquisition, the gels were stained with colloidal Coomassie as previously described for the detection of total protein to view and excise the protein spots that were revealed by this specific staining.

\subsection{Detection of glycoproteins}

Pro-Q Emerald 488 (Molecular Probes) was used to detect glycoproteins in the $2 \mathrm{D}$ gels. Briefly, gels were fixed with two changes of $50 \%$ methanol and 5\% acetic acid for 1 hour per wash, followed by incubation in $1 \%$ periodic acid and $3 \%$ acetic acid for 1 hour to oxidise the glycans on these proteins. After washing in 3\% acetic acid, gels were incubated in a Pro-Q Emerald dye solution for 2 hours in the dark and washed again in 3\% acetic acid to reduce the gel background. All steps were carried out at room temperature with gentle agitation, and the images were captured on a Typhoon Trio system using a $488 \mathrm{~nm}$ laser for excitation and a $520 \mathrm{~nm}$ filter for emission. Following image acquisition, as described above for Pro-Q Diamond staining, the gels were stained with colloidal Coomassie for the visualisation and excision of the protein spots revealed by the glycoprotein-specific staining.

\subsection{In-gel tryptic digestion, sample desalting and MALDI-TOF} TOF spectrometry analysis

Coomassie-, Pro-Q-Emerald- and Pro-Q-Diamondstained spots were excised and digested as previously described with some modifications [14]. Protein spots were excised from the gel and washed with a 1:1 (v/v) $50 \mathrm{mM}$ ammonium bicarbonate ( $\mathrm{pH}$ 8.0)/acetonitrile solution, followed by shaking for $15 \mathrm{~min}$. This washing procedure was repeated with fresh solution until destaining was complete, and the gel was dehydrated by the addition of $200 \mu \mathrm{L}$ of acetonitrile for $5 \mathrm{~min}$. Each sample was then rehydrated with approximately $10 \mu \mathrm{L}$ of ice-cold trypsin solution $(20 \mathrm{ng} / \mu \mathrm{L}$ in 40 $\mathrm{mM}$ ammonium bicarbonate [ $\mathrm{pH} 8.0]$ ) and left on ice for 1 hour. The incubation was allowed to proceed for $16 \mathrm{~h}$ at $37^{\circ}$ C. After the digestion, the peptides were extracted twice by the addition of $30 \mu \mathrm{L}$ of $50 \%$ acetonitrile and $5 \%$ formic acid and transferred to $0.6 \mathrm{~mL}$ tubes. Each sample was concentrated in a vacuum centrifuge to a final volume of 5-10 $\mu \mathrm{L}$. C18 ZipTip micropipette tips (Millipore, Bedford, USA) were used to desalt the peptides. The tips were first activated with acetonitrile and then equilibrated with $0.1 \%$ trifluoroa- cetic acid (TFA) in water. The samples were aspirated and dispensed eight times, and the tips were washed 3-5 times with $0.1 \%$ TFA in water. The peptides retained in the tips were eluted with $1.5 \mu \mathrm{L}$ of $50 \%$ acetonitrile plus $0.1 \%(\mathrm{v} / \mathrm{v})$ TFA in water. Each eluate was immediately spotted on the ABI 192-target MALDI plate (Applied Biosystems, USA) by co-crystallisation with $0.3 \mu \mathrm{L}$ of the a-cyano-4hydroxycinnamic acid matrix (CHCA) $(10 \mathrm{mg} / \mathrm{mL}$ CHCA in $50 \%$ acetonitrile, 0.3 [v/v] TFA in water).

Raw data for protein identification were obtained using the 4700 Proteomics Analyzer (Applied Biosystems, Foster City, CA). Both MS and MS/MS data were acquired in positive and reflectron mode using a neodymium-doped yttrium aluminium garnet (Nd:YAG) laser with a $200-\mathrm{Hz}$ repetition rate. Typically, 1,600 shots were accumulated for spectra in MS mode, while 3,000 shots were accumulated for spectra in MS/MS mode. Up to ten of the most intense ion signals with a signal to noise ratio above 20 were selected as the precursors for MS/MS. External calibration in MS mode was performed using a mixture of four peptides: des-Arg1Bradykinin $(\mathrm{m} / \mathrm{z}=904.47)$, angiotensin $\mathrm{I}(\mathrm{m} / \mathrm{z}=1,296.69)$, Glu1-fibrinopeptide B $(\mathrm{m} / \mathrm{z}=1,570.68)$ and ACTH (18-39) $(\mathrm{m} / \mathrm{z}=2,465.20)$. MS/MS spectra were externally calibrated using known fragment ion masses observed in the MS/MS spectrum of angiotensin I. A search of the MS/MS database was performed against the NCBInr databases using Mascot software (www.matrixscience.com). The search parameters included the allowance of two missed tryptic cleavages and non-fixed modifications of methionine, tryptophan, histidine (oxidation) and cysteine (carbamidomethylation and propionamide). The peptide per sample plate (pps) and peptide per well (ppw) files were generated from the raw (or native) MS data according to the following parameters using Data Explorer Software (Applied Biosystems). For MS1: mass range, 900-4,000 Da; peak density, 15 peaks per $200 \mathrm{Da}$; signal-to-noise ratio, 20; minimum area, $100 \mu \mathrm{m}^{2}$; maximum peaks per spot, 60. For MS2: mass range, 60 or 20 Da for the precursor; peak density, 55 peaks per $200 \mathrm{Da}$; signal-to-noise ratio, 2; minimum area, 10; maximum peaks per precursor, 200. The protein identifications based on the MS/ MS data were validated using Scaffold 2 software (Proteome Software Inc., Portland, OR). The identified proteins were accepted if they possessed $>90.0 \%$ probability, as specified by the Peptide Prophet algorithm [15]. Protein probabilities were assigned by the Protein Prophet algorithm [16].

\subsection{Bioinformatics analysis}

A BLAST search of the genome project databases was performed for Leishmania major (http://www.genedb.org/ Homepage/Lmajor), Trypanosoma brucei brucei (http:// www.genedb.org/Homepage/Tbruceibrucei427) and Homo sapiens (http://www.ncbi.nlm.nih.gov/genome/) to find annotated sequences to all T. cruzi polypeptides identified in this study. Alternatively, to suggest a possible function of the identified hypothetical T. cruzi proteins, another BLAST 
analysis of the whole NCBI database was performed. The function and localisation of the polypeptides was predicted using Gene Ontology (GO, http://www.geneontology.org/), the Kyoto Encyclopaedia of Genes and Genomes (KEGG, http://www.genome.jp/kegg/), Uniprot (http:// www.uniprot.org/) and SwissProt (http://www.expasy.org/ tools/). The high hit sequences (E value $\leq 10^{-50}$ ) of L. major, T. brucei and H. sapiens were aligned to the identified T. cruzi proteins using the multiple sequence alignment (MSA) program T-Coffee (European Bioinformatics Institute, Cambridge, UK). Different sequences ( $\geq 20$ amino acids) observed between the T. cruzi and H. sapiens proteins were classified as non-homologous. Motif prediction analysis was performed using query sequences in NCBI Conserved Domain (http://www.ncbi.nlm.nih.gov/cdd) and ScanProsite tools (http://www.expasy.ch/tools/scanprosite/).

\section{Results}

The protein preparation and two-dimensional electrophoresis conditions for T. cruzi epimastigote samples have been previously established (Menna-Barreto et al., 2010), allowing for high reproducibility and effective spot identification with excellent ion scores. In the colloidal Coomassie-stained gel ( $\mathrm{pH} 4-7$ ), 600 spots were detected and 117 most intensely staining spots were identified as 78 distinct proteins (Figure 1). The complete list of the identified proteins is shown in supplementary Table S1. Between these identified proteins,

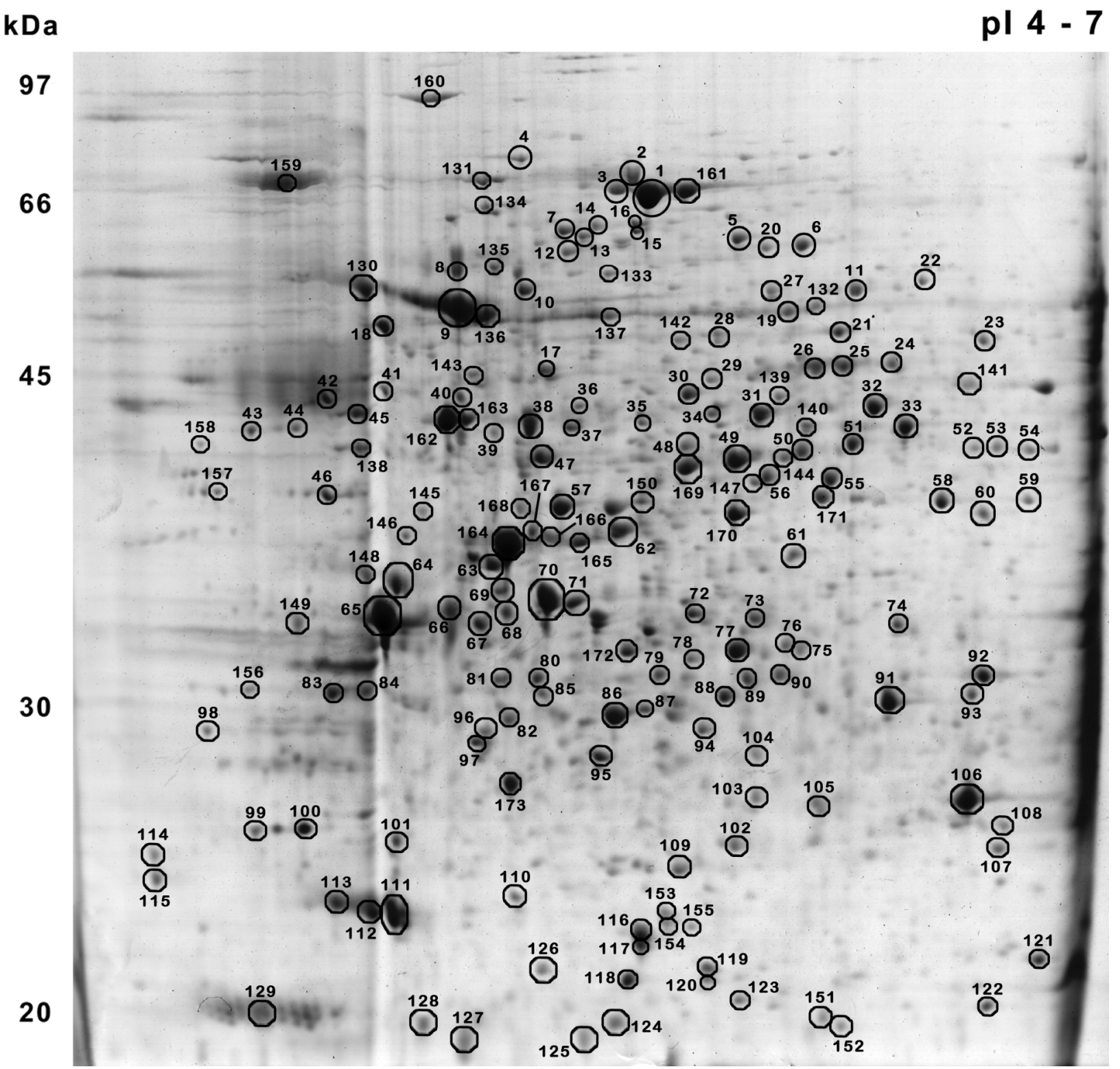

Figure 1. Colloidal Coomassie Blue staining of T. cruzi epimastigotes (Y strain). 2-DE was performed with $500 \mu \mathrm{g}$ of soluble protein using $\mathrm{pH}$ 4-7 IPG strips $(18 \mathrm{~cm})$, followed by $12 \%$ SDS-PAGE. Circles and numbers represent proteins and refer to the spot identification in Table S1 (supplementary data). 
fifteen novel polypeptides not previously addressed in any $T$. cruzi proteome investigation were showed. These proteins are: mitochondrial heat shock, hypothetical proteins 1-6, glycosyl transferase family 2 , metallocarboxypeptidase chain A, protein of unknown function DUF909, unnamed protein product, chaperonin GroEL, twin-arginine translocation pathway signal serine carboxypeptidase, conserved hypothetical protein and Sorting nexin GRD19 homolog. Interestingly, among these molecules, only a few had been described at the protein (3/15) or transcriptional (1/15) level. Most of these proteins were predicted $(10 / 15)$ or even inferred by homology (1/15); this is the first description of their expression in this protozoan.

Here, we showed a map of the post-translational modifications revealed by staining 2-D gels with two different fluorescent dyes for phosphoproteins and glycoproteins. Three hundred and eighty-four phosphoproteins (Pro-QDiamond) and 34 glycoproteins spots (Pro-Q-Emerald) were detected. However, only 34 (Pro-Q-Diamond) and 24 (ProQ-Emerald) spots could be linked to their corresponding protein, resulting in 22 and 15 different proteins, respectively (Figures 2A,B). The complete list of identified proteins is presented in supplementary Table S2. To further investigate the two most common PTMs, phosphorylation and glycosylation, 2D gels were stained with Pro-Q-Diamond and Pro-Emerald, respectively, leading to 31 different identifications, as shown in Table S2. A general evaluation of the previous status of the phosphoproteins and glycoproteins identified here demonstrated a similar percent distribution of proteins described as predicted (11/31), inferred by homology $(8 / 31)$

A

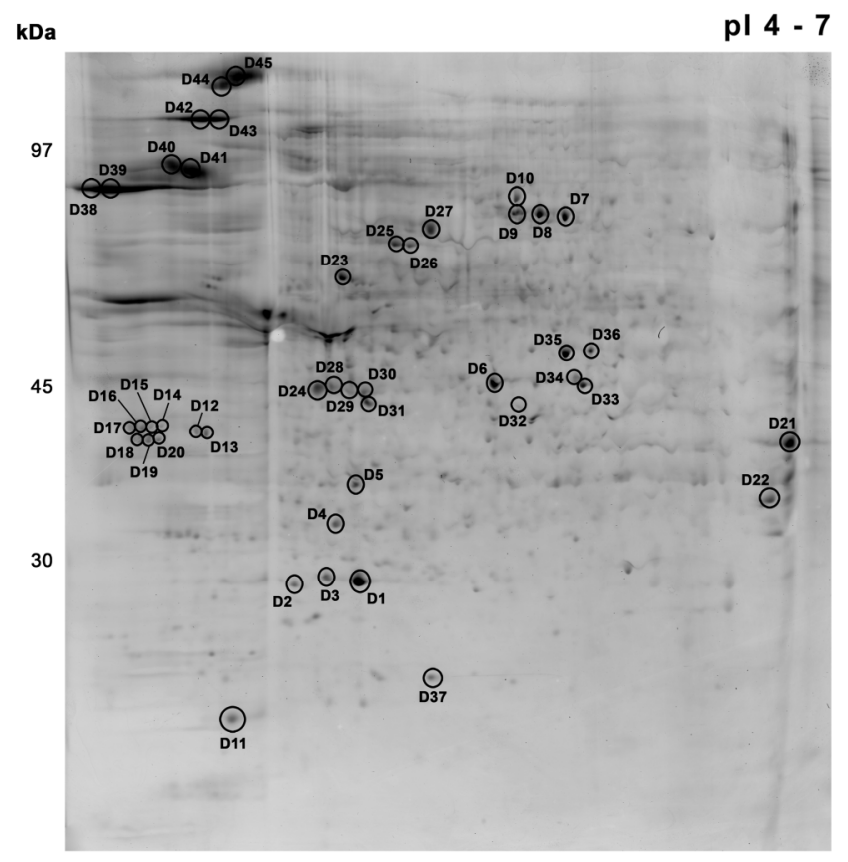

A
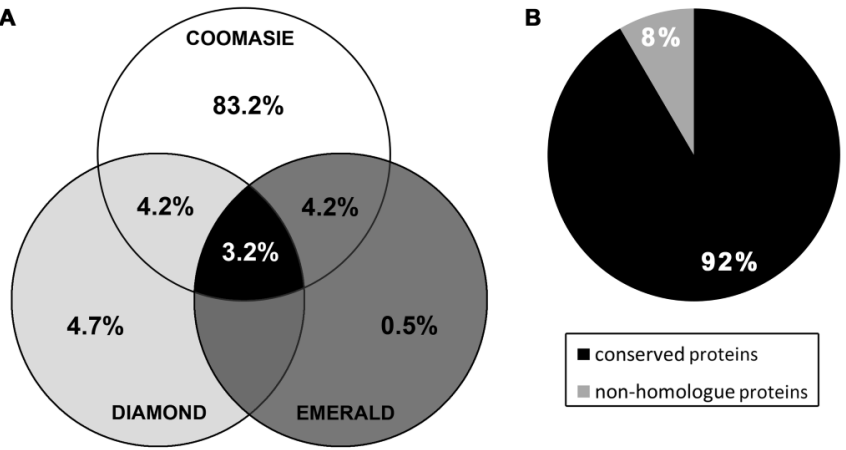

Figure 3. Percentage of protein identification in T. cruzi epimastigote (Y strain) after staining with colloidal Coomassie Blue, Pro-Q-Diamond and Pro-Q-Emerald.

or even detected at the protein (5/31) or transcriptional (5/31) level, reinforcing the necessity and relevance of further high throughput studies on post-translational modifications in protozoan pathogens, including T. cruzi. Surprisingly, some proteins were only identified as phosphoproteins (4.7\%) and glycoproteins (0.5\%) after the use of a specific fluorescent dye (Figure 3A), which could be explained by an increase in colloidal Coomassie sensitivity when the staining was performed after the labelling of the samples with fluorescent dyes (data not shown).

The percentage of peptides identified through each of the three staining protocols varied: colloidal Coomassie staining led to $83.2 \%$ of the peptide identifications, followed by ProQ-Diamond staining (8.9\%) and Pro-Q-Emerald staining $(4.7 \%)$. Only $3.2 \%$ of spots were identified by all of the three protocols (Figure 3). A mascot search of the whole NCBI

B

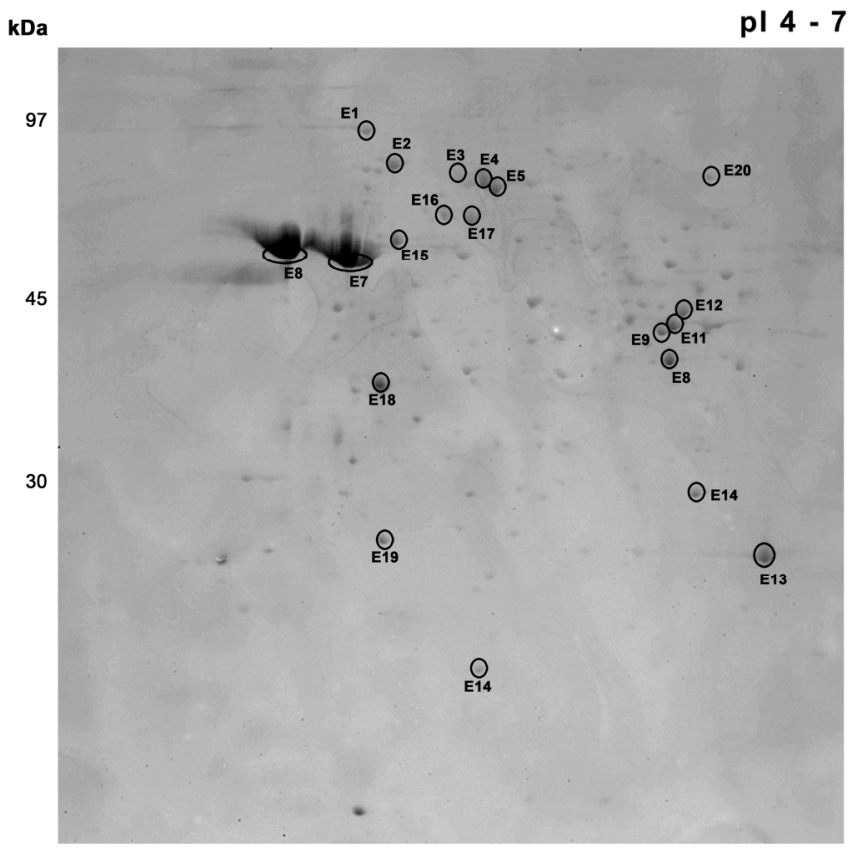

Figure 2. Pro-Q-Diamond (A) and Pro-Q-Emerald (B) staining of T. cruzi epimastigotes (Y strain). 2-DE was performed with $500 \mu \mathrm{g}$ of soluble protein using pH 4-7 IPG strips $(18 \mathrm{~cm}$ ), followed by $12 \%$ SDS-PAGE. Circles and numbers represent proteins and refer to the spot identification in Table S2 (supplementary data). 
database evidenced that $82.2 \%$ of the identified proteins were specific to $T$. cruzi, being a low percentage of identifications $(6.7 \%)$ corresponded to highly conserved proteins that were not associated with any specific organism (data not shown). Compared to the L. major, T. brucei and H. sapiens genome databases, a high percentage of proteins were conserved in trypanosomatids; $92 \%$ of the identified proteins shared high sequence identity or similarity to $H$. sapiens. A low percentage of polypeptides (8\%) did not appear to be homologous to any human peptide sequence (Figure 3B). After the MSA analysis, 21 candidates were selected as potential drug targets by their low similarity to $H$. sapiens proteins. Among those proteins, 10 presented no identity to humans, which two were excluded as potential targets, and another 11 proteins presented promising sequence fragment with no identity to human proteins (Table 1). The motif prediction analysis showed that these sequence fragments represent sites of post -translational modifications, such as phosphorylation, amidation, N-glycosylation and/or N-myristoylation.

Among all of the T. cruzi identifications, 10 hypothetical proteins were found and numbered from 1 to 10 (Figures 1 and 2A, Tables S1 and S2). A BLAST analysis of the whole NCBI database suggested the possible predicted protein functions, as shown in Table S3. Localisation and functional analysis demonstrated that cytosolic proteins were the most abundant (41\%), followed by proteins localised in the mitochondrion (15.4\%), nucleus (9.0\%), glycosome (5.1\%), plasma membrane (3.8\%), endoplasmic reticulum (2.6\%), flagellum (2.6\%), reservosome (1.3\%), cytoskeleton $(1.3 \%)$ and proteasome (1.3\%) under our experimental conditions (Figure 4A). In relation to predicted function, the most common pathway was the protein metabolism pathway, represented by the protein biosynthesis pathway (26.9\%) and degradation pathway (19.2\%), equalling $46.1 \%$ of the total sequences. The other proteins were grouped into pathways including energetic metabolism (23.1\%), motility and intracellular trafficking (9\%), redox balance (2.6\%), cell signalling (2.6\%), lipid metabolism (1.3\%), nucleic acid regulation $(2.6 \%)$ and the polyamine pathway (2.6\%) (Figure 4B).

\section{Discussion}

More than one century after the discovery of Chagas'disease, this illness still represents a serious health problem not only for its endemic region but also for developed countries due to the immigration of infected people. The current treatment of the disease is unsatisfactory, and an efficient prophylactic therapy is required, reinforcing the importance of designing new drug candidates for treatment and identifying alternative drug targets that are specific for the parasite $[3,4,17]$. In this work, we provide additional information on the proteomic map of T. cruzi epimastigotes through the use fluorescent dyes and bioinformatics analysis.

Over the last decade, the proteomic map of different strains of T. cruzi epimastigotes has been extensively investigated, including clone $\mathrm{Dm} 28 \mathrm{c}$ and the sylvan isolates [7-10, 18-23]. About Y strain epimastigotes, the unique large-scale protein investigation was performed in 2008 in order to characterise the molecules involved in the resistance and susceptibility of these parasites to the current chemotherapeutic agent benznidazole. Fifty-five proteins were identified, with approximately $10 \%$ hypothetical proteins [10], similar percentage to that observed in the present work. Here, also employing the 2-D gels followed by MALDI-TOF/TOF analysis, it was identified 78 distinct proteins approximately $30 \%$ more proteins.

Among the 10 trypanosomatid-specific proteins, each one represents a good candidate for drug intervention due to

Table 1. Motif analysis of T. cruzi sequences

\begin{tabular}{ll}
\hline \multicolumn{1}{c}{ PROTEIN } & \multicolumn{1}{c}{ MOTIF FUNCTION a $^{-1}$} \\
\hline heat shock protein 70 & N-myristoylation, phosphorylation \\
thiol transferase Tc52 & phosphorylation \\
ATPase beta subunit & N-myristoylation, phosphorylation \\
serine carboxypeptidase & phosphorylation \\
unnamed protein product & N-myristoylation, phosphorylation \\
arginine kinase & N-glycosylation, phosphorylation \\
cystathionine beta-synthase 6 & amidation, phosphorylation \\
dihydrolipoamide acetyltransferase & phosphorylation \\
aminopeptidase & N-myristoylation, phosphorylation \\
arginase superfamily protein & N-myristoylation, phosphorylation \\
RNA-binding protein RGGm & N-myristoylation, phosphorylation, amidation \\
\hline
\end{tabular}

${ }^{\text {a }}$ Sequences of $>20$ amino acids that are absent in human homologues. 
A
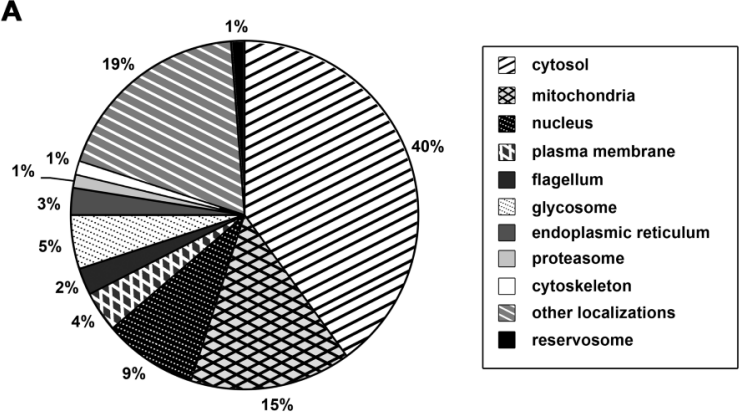

B

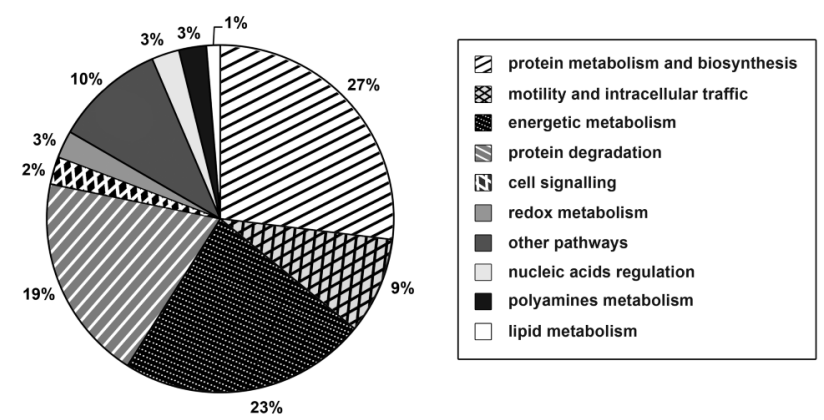

Figure 4. Localisation and functional classification of T. cruzi epimastigote (Y strain) proteins. (A) Organellar localisation of all proteins identified in this study. (B) Known or predicted function of each identified protein.

their inhibition could lead to the parasite death by a different pathway. Trypanothione synthetase is a crucial molecule in the main anti-oxidant pathway in trypanosomatids [24], related to ROS balance. 24-C-methyltransferase participates in ergosterol biosynthesis [25], directly involved in the parasite's plasma membrane fluidity. Calpain is a protease associated to the protozoa differentiation and infectivity $[26,27]$. A predicted dehydrogenase was better characterised in 2006 as prostaglandin F2 alpha synthase [28], being related to detoxification processes. Phosphoenolpyruvate carboxykinase is a key enzyme in gluconeogenesis [29]. Nucleoside phosphorylase participates in purine metabolism, being fundamental for nucleoside recovery after nucleotide degradation $[30,31]$. HslVu is a subunit of a large complex that degrades misfolded proteins called the proteasome [32]. I/6 autoantigen is a calcium-binding microtubule-associated protein responsible for crosslinking microtubule filaments and involved mitosis, vesicle trafficking and other events [33].

The last two candidates are involved in bioenergetics and for different reasons are not promising targets. P28 is a predicted pyruvate kinase superfamily protein that presents almost a half of the identical sequence of pyruvate kinase. The high similarity of pyruvate kinase to the corresponding mammalian protein led us to exclude P28 as a potential molecular target for novel trypanocidal drugs. Cytochrome c oxidase is a fundamental enzyme of respiratory electron transport chain [34]. Despites the differences between human and T. cruzi complex IV, the presence of alternative oxidases in the parasite [35], suggests that this protein is not a good target for drug design.
Comparison of the identified T. cruzi epimastigote proteins with other trypanosomatid and human sequences allowed for the selection of 21 proteins as potential drug targets, among which 10 displayed no hits in a BLAST analysis against human database and these trypanosomatid-specific proteins will further discussed here. The other 11 proteins presented stretches of more than 20 amino acids that did not align to the respective human sequences in the MSA, but the possibilities of misalignment due to the presence of multimeric proteins indicated that better algorithms must be used in order to increase the confidence.

PTMs of proteins are well characterised to be functionally important for various physiological and pathological processes, such as signal transduction and cellcell recognition [36]. Until this study, Y strain epimastigotes' glycoprotein content has not been assessed. In 2006, the glycoproteome of trypomastigotes (Brazil strain) was published [37], demonstrating the presence of 31 glycosylated proteins, absent here. Because glycosylation could be fundamental for host cell recognition of the protozoa, it is expected that the infective parasite form presented a different pool of molecules in the relation to the insect form. Nakayasu and co-workers (2009) preliminarily assessed phosphoproteome of Y strain epimastigotes by LC-MS/MS [38], identifying 107 proteins that was not found here. Surprisingly, 19 of the phosphoproteins identified here by fluorescent staining of $2 \mathrm{D}$ gels and MALDI-TOF/TOF were not in the previous $\mathrm{Y}$ strain identification list. These molecules are heat shock-like 85, alpha tubulin, peroxiredoxin, paraflagellar rod protein 1D, glutamine synthetase, actin, pyrroline-5carboxylate synthetase, 14-3-3 protein, chain A of spermidine synthase, enolase 1, eukaryotic initiation factor $5 \mathrm{a}$, chaperonin containing T-complex protein, $80 \mathrm{kDa}$ prolyl oligopeptidase, aminoacylase, proteasome beta 3 subunit and hypothetical proteins 7, 8, 9 and 10 .

\section{Concluding remarks}

Despite many previous efforts to describe the proteome of epimastigotes, new molecules are still being identified in this proliferative form of T. cruzi. Our data could provide new insights into the signalling networks of this protozoan, supplying additional information about its cell biology. Here, it was identified 8 promising good candidates for drug intervention, being necessary a validation in the mammalian forms of parasite.

\section{Supplementary material}

Supplementary data and information is available at: http:// www.jiomics.com/index.php/jio/rt/suppFiles/107/0.

Supplementary Material includes Tables S1, S2, S3 and MS/MS spectra data.

\section{Acknowledgments}


We are very thankful to Nadja Oliveira and Thabata Duque for their help in the comparative proteome analysis. This work was supported with grants from CNPq, FAPERJ, PDTIS/FIOCRUZ and FIOCRUZ.

\section{References}

1. A.C. Aufderheide, W. Salo, M. Madden, J. Streitz, J. Buikstra F. Guhl, B. Arriaza, C. Renier, L.E. Jr Wittmers, G. Fornaciari, M. Allison, PNAS 101 (2004) 2034-2039.

2. S.L. De Castro, D.G. Batista, M.M. Batista, W. Batista, A. Daliry, E.M. de Souza, R.F.S. Menna-Barreto, G.M. Oliveira, K. Salomão, C.F. Silva, P.B. Silva, M.N. Soeiro, Mol. Biol. International (2011), 306928.

3. J.R. Coura, S.L. De Castro, Mem. Inst. Oswaldo Cruz 97 (2002) 3-24.

4. M.N. Soeiro, S.L. De Castro, Exp. Opinion Ther. Targets 13 (2009) 105-121.

5. C. Clayton, M. Shapira, Mol. Biochem. Parasitol. 156 (2007) 93-101.

6. F.B. Holetz, L.R. Alves, C.M. Probst, B. Dallagiovanna, F.K Marchini, P. Manque, G. Buck, M.A. Krieger, A. Correa, S. Goldenberg, FEBS J. 277 (2010) 3415-3426.

7. M. Ferella, D. Nilsson, H. Darban, C. Rodrigues, E.J. Bontempi, R. Docampo, B. Andersson, Proteomics 8 (2008) 27352749.

8. A. Parodi-Talice, R. Durán, N. Arrambide, V. Prieto, M.D. Piñeyro, O. Pritsch, A. Cayota, C. Cerveñansky, C. Robello, Int. J. Parasitol. 34 (2004) 881-886.

9. J.A. 3rd Atwood, D.B. Weatherly, T.A. Minning, B. Bundy, C. Cavola, F.R. Opperdoes, R. Orlando, R.L. Tarleton, Science 309 (2005) 473-476.

10. H.M. Andrade, S.M. Murta, A. Chapeaurouge, J. Perales, P. Nirdé, A.J. Romanha, J. Proteom. Res. 7 (2008) 2357-2367.

11. R.F. Menna-Barreto, D.G. Beghini, A.T. Ferreira, A.V. Pinto, S.L. De Castro, J. Perales, J. Proteomics 73 (2010) 2306-2315.

12. J.A. Urbina, Acta Tropica 115 (2010) 55-68.

13. R. Devera, O. Fernandes, J.R. Coura, Mem. Inst. Oswaldo Cruz 98 (2003) 1-12.

14. A. Shevchenko, O.N. Jensen, A.V. Podtelejnikov, F. Sagliocco, M. Wilm, O. Vorm, P. Mortensen, A. Shevchenko, H. Boucherie, M. Mann, PNAS 93 (1996) 14440-14445.

15. A. Keller, A.I. Nesvizhskii, E. Kolker, R. Aebersold, Analyt. Chem. 74 (2002) 5383-5392.

16. A.I. Nesvizhskii, A. Keller, E. Kolker, R. Aebersold, Analyt. Chem. 75 (2003) 4646-4658.

17. J.C.P. Dias, Cadernos de Saúde Pública 23 (2007) 13-22.
18. J. Paba, J.M. Santana, A.R. Teixeira, W. Fontes, M.V. Sousa, C.A. Ricart, Proteomics 4 (2004) 1052-1059.

19. E.M. Cordero, E.S. Nakayasu, L.G. Gentil, N. Yoshida, I.C. Almeida, J.F. da Silveira, J. Proteom. Res. 8 (2009) 3642-3652.

20. C.L. Sodré, A.D. Chapeaurouge, D.E. Kalume, L. MendonçaLima, J. Perales, O. Fernandes, Arch. Microbiol. 191 (2009) 177-184.

21. S.A. Kikuchi, C.L. Sodré, D.E. Kalume, C.G. Elias, A.L. Santos, M.N. Soeiro, M. Meuser, A. Chapeaurouge, J. Perales, O. Fernandes, Exp. Parasitol. 126 (2010), 540-551.

22. E.S. Nakayasu, T.J. Sobreira, R.Jr Torres, L. Ganiko, P.S. Oliveira, A.F. Marques, I.C. Almeida, J. Proteome Res. 11 (2011) 237-246.

23. D. Pérez-Morales, H. Lanz-Mendoza, G. Hurtado, R. Martínez-Espinosa, B. Espinoza, J. Biomed. Biotech. (2012) 902803.

24. F. Irigoín, L. Cibils, M.A. Comini, S.R. Wilkinson, L. Flohé, R. Radi, Free Rad. Biol. Med. 45 (2008) 733-742.

25. J.A. Urbina, International Symposium on the Centennial of the Discovery of Chagas' Disease. Rio de Janeiro, 2009.

26. V. Ennes-Vidal, R.F. Menna-Barreto, A.L. Santos, M.H. Branquinha, C.M. d'Avila-Levy, J. Antimicr. Chemother. 65 (2010) 1395-1398.

27. V. Ennes-Vidal, R.F. Menna-Barreto, A.L. Santos, M.H. Branquinha, C.M. d'Avila-Levy, PLoS One 6 (2011) e18371.

28. S.M. Murta, M.A. Krieger, L.R. Montenegro, F.F. Campos, C.M. Probst, A.R. Avila, N.H. Muto, R.C. Oliveira, L.R. Nunes, P. Nirdé, O. Bruna-Romero, S. Goldenberg, A.J. Romanha, Mol. Biochem. Parasitol. 146 (2006), 151-162.

29. S. Trapani, J. Linss, S. Goldenberg, H. Fischer, A.F. Craievich, G. Oliva, J. Mol. Biol. 313 (2001) 1059-1072.

30. R.L. Miller, C.L. Sabourin, T.A. Krenitsky, Biochem. Pharmacol. 36 (1987) 553-560.

31. R.G. Silva, M.J. Vetticatt, E.F. Merino, M.B. Cassera, V.L. Schramm, J. Am. Chem. Soc. 133 (2011) 9923-9931.

32. C. Gille, A. Goedel, C. Schloetelburg, R. Preißner, P.M. Kloetzell, U.B. Gobel, C. Frommell, J. Mol. Biol. 326 (2003) 1437-1448.

33. E. Detmer, A. Hemphill, N. Müller, T. Seebeck, Eur. J. Cell Biol. 72 (1997) 378-384.

34. P. Brzezinski, R.B. Gennis, J. Bioenerg. Biomemb. 40 (2008) 521-531.

35. J.J. Wen, N.J. Garg, Antioxid. Red. Sign. 12 (2010) 27-37.

36. M. Mann, O.N. Jensen, Nature Biotech. 21 (2003) 255-261.

37. J.A. 3rd Atwood, T. Minning, F. Ludolf, A. Nuccio, D.B. Weatherly, G. Alvarez-Manilla, R. Tarleton, R. Orlando, J. Proteome Res. 5 (2006) 3376-3384.

38. E.S. Nakayasu, M.R. Gaynor, T.J. Sobreira, J.A. Ross, I.C. Almeida, Proteomics 9 (2009) 3489-3506. 